\title{
In situ Synthesis of DNA Microarray on Functionalized Cyclic Olefin Copolymer Substrate
}

\author{
Ishtiaq Saaem, ${ }^{\dagger,}$ ${ }^{\dagger}$ Kuo-Sheng Ma, ${ }^{\dagger,}$ Alexandria N. Marchi, ${ }^{\dagger}$ Thomas H. LaBean, ${ }^{\dagger, \S, \|}$ and \\ Jingdong Tian*, ${ }^{*}$,
}

Department of Biomedical Engineering, Institute for Genome Sciences and Policy, Department of Computer

Science, and Department of Chemistry, Duke University, Durham, North Carolina 27708

\begin{abstract}
Thermoplastic materials such as cyclic-olefin copolymers (COC) provide a versatile and cost-effective alternative to the traditional glass or silicon substrate for rapid prototyping and industrial scale fabrication of microdevices. To extend the utility of $\mathrm{COC}$ as an effective microarray substrate, we developed a new method that enabled for the first time in situ synthesis of DNA oligonucleotide microarrays on the $\mathrm{COC}$ substrate. To achieve high-quality DNA synthesis, $\mathrm{a} \mathrm{SiO}_{2}$ thin film array was prepatterned on the inert and hydrophobic COC surface using RF sputtering technique. The subsequent in situ DNA synthesis was confined to the surface of the prepatterned hydrophilic $\mathrm{SiO}_{2}$ thin film features by precision delivery of the phosphoramidite chemistry using an inkjet DNA synthesizer. The in situ $\mathrm{SiO}_{2}-\mathrm{COC}$ DNA microarray demonstrated superior quality and stability in hybridization assays and thermal cycling reactions. Furthermore, we demonstrate that pools of high-quality mixed-oligos could be cleaved off the $\mathrm{SiO}{ }_{2}-\mathrm{COC}$ microarrays and used directly for construction of DNA origami nanostructures. It is believed that this method will not only enable synthesis of high-quality and low-cost COC DNA microarrays but also provide a basis for further development of integrated microfluidics microarrays for a broad range of bioanalytical and biofabrication applications.
\end{abstract}

KEYWORDS: DNA microarray $\bullet$ DNA synthesis $\bullet$ COC $\bullet$ synthetic biology $\bullet$ microfluidics $\bullet$ DNA origami

\section{INTRODUCTION}

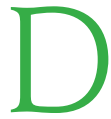
NA microarrays have become a very important and versatile tool for a wide range of applications ranging from high-throughput genomics, proteomics, to drug discovery, biodetection, and synthetic biology (1-5). In addition to on-chip assays, in situ synthesized oligonucleotide arrays can also be used to generate pools of mixed oligos for off-chip use as oligo libraries or gene building blocks (6-9).

Glass and silicon have traditionally been used for microarray fabrication due to their excellent physical properties and diverse surface functionalization chemistries (4). Glass offers additional benefits thanks to its superior optical properties (i.e., high transparency and low intrinsic fluorescence). Recently, thermoplastic materials such as cyclic olefin copolymers (COC), poly(methylmethacrylate) (PMMA), and polycarbonate (PC) have been tested as low-cost alternative array substrates, especially for making devices involving integration of microarrays with microfluidics (10-15). Combining microarrays with microfluidics offers significant advantage in portability, analysis speed, sensitivity, and

\footnotetext{
* Corresponding author. E-mail: jtian@duke.edu. Tel: (919) 684-3494. Fax (919) 684-4488

Received for review December 14, 2009 and accepted January 29, 2010

† Department of Biomedical Engineering, Duke University.

* Institute for Genome Sciences and Policy, Duke University.

$\S$ Department of Computer Science, Duke University.

" Department of Chemistry, Duke University.

DOI: $10.1021 / a m 900884 b$

( 2010 American Chemical Society
}

conservation of precious biological analytes (16-19). These thermoplastic materials are inexpensive, durable, and can be easily embossed or molded for rapid prototyping and mass production of microdevices (20-23). COC is particularly attractive because of a combination of desirable characteristics, including excellent optical and UV transparency, low autofluorescence, low density, high stiffness, high glasstransition temperature $\left(T_{\mathrm{g}}\right.$, up to $\left.180^{\circ} \mathrm{C}\right)$, low moisture adsorption, and resistance to organic solvents (10, 24-26).

Despite the potentially broad and significant applications, in situ synthesis of DNA microarray on COC has been challenging and yet to be demonstrated. COC presents a chemically inert and highly hydrophobic surface, making in situ DNA synthesis difficult. A number of plastic surface treatment methods have been developed for generating surface functional groups and reducing hydrophobicity, such as photo grafting or treatment with oxygen plasma, UV/ ozone or sodium hydroxide $(10,27-35)$. These methods have been used for immobilization of presynthesized DNA probes on COC surface (10-13, 36-42), but have not been proven readily applicable for in situ DNA synthesis on COC.

We have recently described the fabrication and characterization of a $\mathrm{SiO}_{2}-\mathrm{COC}$ hybrid material, which makes a suitable substrate for potential bioMEMS applications (43). On the basis of this concept, here we report in situ synthesis of high-quality oligonucleotide arrays on prepatterned $\mathrm{SiO}_{2}-\mathrm{COC}$ slides. In situ DNA synthesis was achieved with a custom piezoelectric inkjet system using the phosphoramidite chemistry. We demonstrated that the in situ COC oligonucleotide arrays were useful for nucleic acid hybridiza- 
tion assays and enzymatic reactions such as PCR. When synthesized with a cleavable linker, the oligos could be eluted from the chip and used directly for constructing DNA origami nanostructures.

\section{MATERIALS AND METHODS}

Materials. Cyclic olefin copolymers (COC) resins (Topas 6015S-04) and COC plates (Topas 6015, 6" $\times 6^{\prime \prime}$ ) were purchased from TOPAS Advanced Polymers (Florence, KY). Polydimethylsiloxane was purchased from Dow Corning (Midland, MI). Positive photoresist Shipley 1813 and developer were purchased from MicroChem Corp. (Newton, MA). Nucleoside phosphoramidites (Pac-dA-CE, Ac-dC-CE, iPr-Pac-dG-CE and dTCE), 5-ethylthio-1H-tetrazole, Cap Mix A (5\% Phenoxyacetic anhydride in THF), and oxidizer (0.02 $\mathrm{M}$ iodine in pyridine/ tetrahydrofuran/water) were purchased from Glen Research (Sterling, VA). Phosphoramidites and 5-ethylthio-1 H-tetrazole were dissolved at 0.25 and $0.625 \mathrm{M}$, respectively, in a mixture of $50 \%$ 3-methoxypropionitrile and $50 \%$ glutaronitrile (Sigma, St. Louis, MO). The solvents were dried for 2 days on molecular sieves before use. Synthesis-grade anhydrous acetonitrile, Cap Mix B (n-methylimidazole in THF) and deblocking solution (trichloroacetic acid in DCM) were purchased from Azco Biotech (San Diego, CA)

Patterning COC Slides and Functionalization. Prior to silica deposition, an array of microwells with bare COC bottom was created in a layer of photoresist which was spin-coated on the COC slide surface using standard photolithography techniques. Deposition of silica within the microwells was then performed as described elsewhere (43). After deposition, the remaining photoresist was stripped by sonication in acetone. All patterned samples were immersed into a $1: 1 \mathrm{H}_{2} \mathrm{SO}_{4}: \mathrm{H}_{2} \mathrm{O}_{2}$ solution for 15 min to remove resist residue and finally rinsed with deionized water and blown dry. For a detailed experimental description, please refer to the Supporting Information.

Patterned substrates to be used for oligo cleavage and harvesting were placed in a $2 \%(\mathrm{v} / \mathrm{v}) \mathrm{N}$-(3-triethoxysilylpropyl)4-hydroxy-butyramide (Gelest, Inc., Morrisville, PA) solution in $95 \%$ ethanol overnight with stirring in a nitrogen-purged cabinet. The samples were then rinsed and sonicated for $30 \mathrm{~s}$ in ethanol, and then baked in a preheated oven $\left(110^{\circ} \mathrm{C}\right)$ for $3 \mathrm{~h}$. Samples were stored desiccated until ready for use.

In situ Oligonucleotide Synthesis. In situ DNA microarray synthesis utilized standard phosphoramidite chemistry with a custom-built piezoelectric inkjet platform based on a previously described system (44). A 1:1 mixture of methyl glutaronitrile (MGN) and 3-methoxypropionitrile (3MP) was used as solvent instead of volatile acetonitrile. Four channels on the printhead delivered phosphoramidite monomers (A, T, G, and C) and one delivered an activator (ethylthiotetrazole). Bulk reagents (i.e., oxidizer, deprotection acid, acetonitrile, Cap A, Cap B) and waste are stored in glass bottles with GL-45 screw-top caps. Bulk reagents were controlled using PTFE solenoid valves leading to delivery lines colocated near the printhead (or to the slide holder for the waste line). Approximately $0.5 \mathrm{~mL}$ of each bulk reagent was added at a time, which was enough to cover the slide surface. An optical system is also colocated near the printhead, which captures high contrast images of printed droplets using a CCD detector and collimated LED light, enabling alignment of the printhead with a slide's silica features.

Each cycle of synthesis includes (1) printing, (2) washing, (3) capping, (4) washing, (5) oxidation, (6) washing, (7) detritylation, and (8) washing. Slides were blown dry after each step except printing by a six-jet nitrogen manifold controlled by a solenoid valve. The first step of the reaction consists of phosphoramidite monomer printing followed by tetrazole printing, and incubation for $2 \mathrm{~min}$. On nonsilanized arrays where coupling occurred with surface hydroxyls directly on the silica, the first base was reprinted 4 times with $1 \mathrm{~h}$ incubation each time. Oxidization and capping were carried out for $30 \mathrm{~s}$, whereas detritylation lasted $10 \mathrm{~s}$. Washing after detritylation was done for $4 \mathrm{~s}$ but after every other step was for only $2 \mathrm{~s}$.

After synthesis, the COC slides were removed from the synthesis platform and rinsed with acetonitrile and then $95 \%$ ethanol. The base-protecting groups were removed by $2 \mathrm{~h}$ incubation in EDA/EtOH (anhydrous, 1:1 v/v) at room temperature. The deprotected slides were rinsed five times with deionized water, dried, and stored in a desiccator.

Staining and Hybridization Procedure. After synthesis and deprotection, the COC slide was placed in a $3 \%(\mathrm{w} / \mathrm{v})$ Pluronic F108 solution for 10 min (45). Pluronic (BASF, Research Triangle Park, NC, USA), a triblock copolymer consisting of PEO-PPOPEO units, hydrophobically adsorbs onto the background $\mathrm{COC}$ via its PPO unit while the hydrophilic PEO chains minimize nonspecific adsorption. Slides were then blown dry.

To stain with SYBR Gold, we dissolved $1 \mu \mathrm{L}$ of SYBR gold (S11494, Invitrogen) in $10 \mathrm{~mL}$ of Tris/Borate/EDTA (TBE) buffer. Slides carrying synthesized oligos and blocked with Pluronic were incubated for $15 \mathrm{~min}$ in the SYBR gold solution. Slides were then rinsed in DI water three times by soaking them in a staining jar at room temperature (5 min each time).

For hybridization experiments, the target was dissolved in a $6 \times$ SSPE solution ( $100 \mu \mathrm{L}, 1 \mathrm{M} \mathrm{NaCl}, 66 \mathrm{mM}$ sodium phosphate, $6 \mathrm{mM}$ EDTA, pH 7.4) and heated at $95^{\circ}$ for $5 \mathrm{~min}$. The hybridization experiment was performed using a Biorad hybridization chamber at room temperature for $2 \mathrm{~h}$. After hybridization, the slide was washed twice ( 5 min each) with the $6 \times$ SSPE buffer, once (30 s) with $2 \times$ SSPE, $0.1 \%$ SDS, and finally quickly dipped into $0.2 \times$ SSPE before being dried and scanned.

After recording the fluorescence from the initial hybridization, to ascertain the stability of oligos on nonsilanized slides, we loaded synthesized slides with Phusion High-Fidelity PCR Master Mix (that contains Phusion High-Fidelity DNA Polymerase, nucleotides and an optimized reaction buffer including $\mathrm{MgCl}_{2}$ ) (Finnzymes, Espoo, Finland) with $0.1 \%$ BSA (Promega, Madison, WI, USA) and $0.1 \%$ Tween-20 (Sigma) using a hybridization chamber. Slides were then thermocycled $\left(94{ }^{\circ} \mathrm{C}\right.$ for 9 min and 50 cycles of $94{ }^{\circ} \mathrm{C}$ for $45 \mathrm{~s}, 65^{\circ} \mathrm{C}$ for $3 \mathrm{~min}$ and $72{ }^{\circ} \mathrm{C}$ for 4 min) using a Master Thermocycler (Eppendorf). Following this treatment, slides were rinsed with water and rehybridized.

Elution of Oligonucleotides and Formation of DNA Origami. Oligos used for gel analysis and DNA origami construction were cleaved from patterned samples that had been silanized prior to synthesis. Slides were affixed with a Biorad hybridization chamber and loaded with $300 \mu \mathrm{L}$ of $\mathrm{NH}_{4} \mathrm{OH}$. Once loaded, the slides were placed under $\sim 80$ psi of pressure using a pneumatic clamp assembly (Biolytic Lab Performance Inc., Newark, CA) for $18 \mathrm{~h}$ at room temperature. The eluate was collected from slides and dried down in a speed vacuum centrifuge (Eppendorf, Hamburg, Germany) and the precipitate was resuspended in sterile Milli-Q water (where the precipitates from three slides were pooled and resuspended in $5 \mu \mathrm{L}$ ).

For the formation of tall rectangle and sharp triangle DNA origami nanostructures, M13 single-stranded phage DNA (Bayou Biolabs) and the synthesized staple strands for the tall rectangle or sharp triangle were mixed together with a 10-fold molar excess of staples (46). Anneals were performed at $5 \mu \mathrm{M}$ concentration of scaffold in $1 \times \mathrm{TAE} / \mathrm{Mg}^{2+}$ buffer $(40 \mathrm{mM}$ Tris$\mathrm{HCl}$ (pH 8.0), $20 \mathrm{mM}$ acetic acid, $2 \mathrm{mM}$ EDTA, and $12.5 \mathrm{mM}$ magnesium acetate) and cooled by $1{ }^{\circ} \mathrm{C} / \mathrm{min}$ from 94 to $20^{\circ} \mathrm{C}$. The samples were incubated at $4{ }^{\circ} \mathrm{C}$ for at least $2 \mathrm{~h}$ before AFM imaging. Staple strand sequences are given in Supporting Information. 


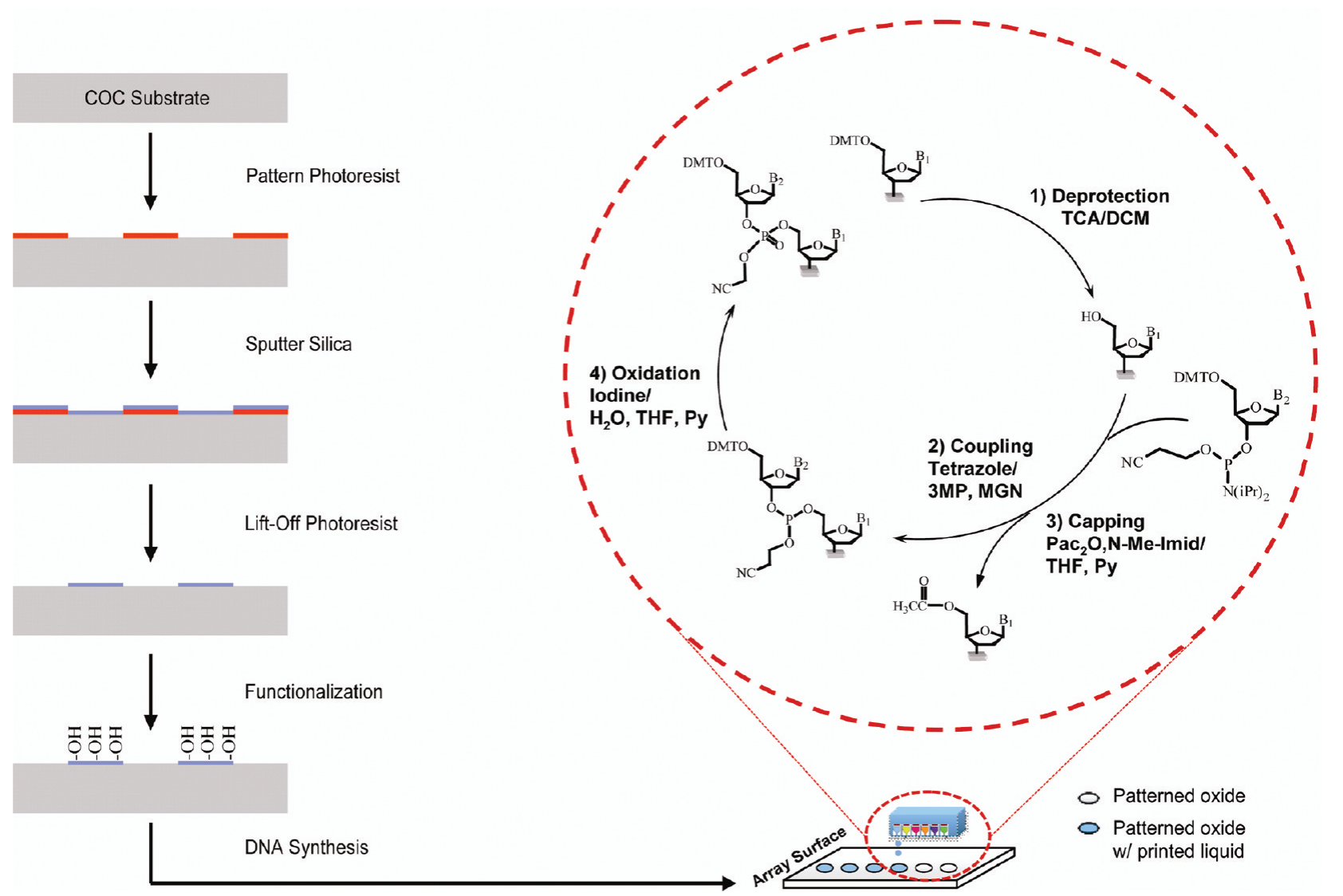

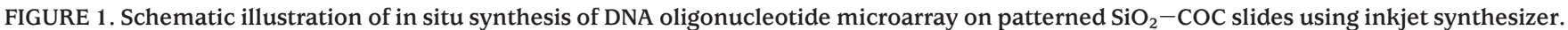
The left panel depicts the fabrication process of the patterned $\mathrm{SiO}_{2}-\mathrm{COC}$ slide via photolithography and RF sputtering techniques; the right panel illustrates the phosphoramidite chemistry used for in situ oligonucleotide microarray synthesis on $\mathrm{SiO}_{2}-\mathrm{COC}$ slides.

\section{RESULTS}

We used a custom-built inkjet system to synthesize oligonucleotide microarrays on prepatterned $\mathrm{SiO}_{2}-\mathrm{COC}$ slides. The patterned $\mathrm{SiO}_{2}-\mathrm{COC}$ slides were prepared following the procedure as illustrated in Figure 1. Prior to silica deposition, an array of microwells with bare COC bottom was created in a layer of photoresist that was spin-coated on the COC slide surface using standard photolithography techniques. After sputter deposition of silica thin film, the photoresist was removed by acetone and a silica thin film pattern was left behind only on the previously exposed microwell bottom. Subsequently, oligos were synthesized on the hydrophilic surface of prepatterned silica features using a custom-built inkjet DNA synthesizer. Oligos were either synthesized directly on native silanols $(-\mathrm{OH})$ presented on the silica arrays after hydroxylating with Piranha solution or on arrays silanized with a cleavable linker.

Proper formation of the silica arrays on COC was confirmed by scanning electron microscopy (SEM) and atomic force microscopy (AFM) (Figure 2). Figure 2A and its highermagnification inset show that features, with a $50 \mu \mathrm{m}$ diameter and $100 \mu \mathrm{m}$ pitch, had well-defined and smooth boundaries. The thickness of the deposited silica thin film was approximately $250 \mathrm{~nm}$ as determined by AFM height profiling. The images were derived from an array of forty thousand features patterned on a COC slide. The lack of any defects or missing features indicated that the bonding between silica and COC was sufficiently strong to sustain
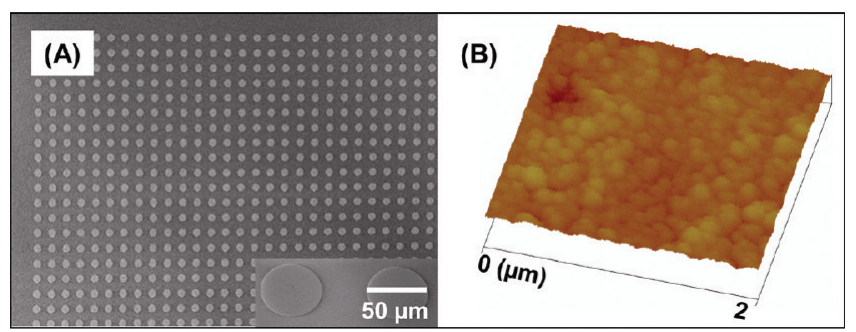

FIGURE 2. Characterization of patterned $\mathrm{SiO}_{2}$ features on the COC slide surface. (A) SEM image showing a subset of an array of forty thousand $\mathrm{SiO}_{2}$ features. The inset presents a higher magnification image of the features. (B) AFM image of the $\mathrm{SiO}_{2}$ thin film surface.

extensive sonications during the microfabrication process. It was also noticed that the silica-COC bonding was able to withstand repeated attempts to dislodge them by sticking and peeling of an adhesive tape from the slide surface.

AFM analysis indicated that the surface of the sputtered silica thin film had flatness comparable to that of COC or standard glass slide surface (Figure $2 \mathrm{~B}$ ). The average roughness $\left(R_{\mathrm{a}}\right)$ over a $4 \mu \mathrm{m}^{2}$ area was $3.75 \pm 0.53 \mathrm{~nm}$. The silica features were able to constrain liquid via differential surface wettability between the deposited silica and the COC background, thereby effectively forming an array of hydrophilic picoliter wells. The silica features on COC also demonstrated excellent chemical compatibility and remained intact after repeated exposures to DNA chemical synthesis reagents 


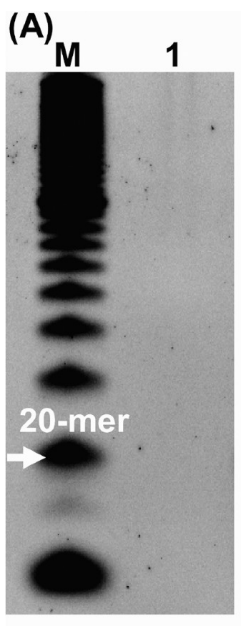

(B)

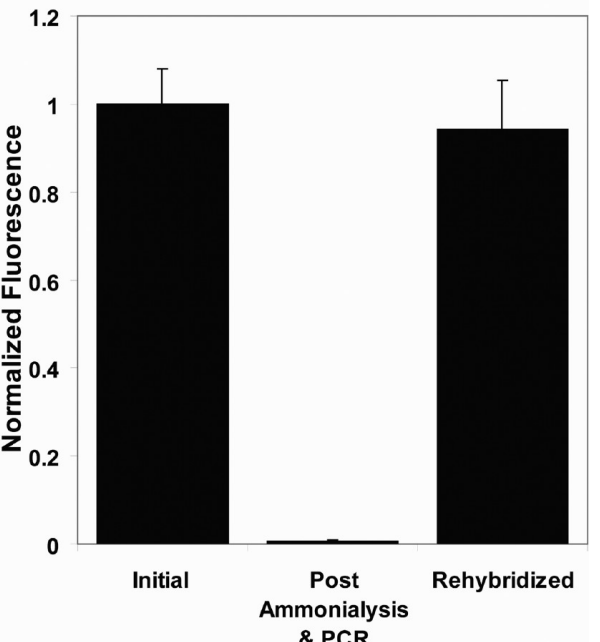

\& PCR

FIGURE 5. Stability of oligonucleotides synthesized on nonsilanized arrays. (A) Slides synthesized with Probe A and subjected to $18 \mathrm{~h}$ of ammonialysis at RT did not release detectable amount of DNA as indicative by the lack of a 20 -mer band in Lane 1. Lane M, 10-bp ladder. (B) Slides synthesized with Probe B were subjected to $18 \mathrm{~h}$ of ammonialysis at RT, followed by a 50-cycle thermocycling. Hybridization signal afterward showed no significant difference from untreated slides.

detectable amount of oligos had been cleaved from the slides. In Figure 5B, Probe B was synthesized on nonsilanized slides, deprotected, and hybridized with Cy5-labeled target. Postscanning, they were ammonialysed for $18 \mathrm{~h}$. At this point, to determine stability of the silica functionalization and oligos to thermal cycling conditions, the same slides were loaded with Phusion High-Fidelity PCR Master Mix and thermocycled for 50 cycles as described in the Methods. Stability of the oligos and their attachment to the slide were clear, as rehybridization after the PCR thermocycling resulted in approximately $96 \%$ of the initial fluorescence.

With a cleavable linker, such as $\mathrm{N}$-(3-triethoxysilylpropyl)4-hydroxy-butyramide, the in situ synthesized oligos were able to be cleaved off the chip and harvested for potential applications. In this study, we wanted to determine if chipsynthesized oligos could be used for construction of DNA origami nanostructures. We first examined the size and quantity of the oligo pools synthesized and harvested from the silanized slides (see the Supporting Information for oligo sequences). In one experiment, 225 unique oligos of mixed lengths were synthesized. The total products from 3 replicate sets of slides were pooled together and dried down. The precipitate was then resuspended and analyzed by $15 \%$ polyacrylamide TBE-Urea gel electrophoresis with SYBR Gold staining. In this pool of mixed oligos, greater than $85 \%$ were 32-mers, and about $12 \%$ were 36-mers. Accordingly, a broadband representing the mixed oligos was seen in Figure $6 \mathrm{~A}$, lane 1 . We calculated on the basis of the UV spectrophotometer measurements that approximately $70 \mathrm{pmol}$ of DNA was collected from the 3 replicate sets of slides, which corresponded to an average oligo density of $\sim 0.13-0.2$ $\mathrm{pmol} / \mathrm{mm}^{2}$ in a spot.

The sequences of these oligos had been designed to serve as staples to fold single-stranded M13 DNA scaffolds into nanostructures resembling a tall rectangle or sharp triangle (46). Using two separate pools of oligos harvested from the COC slides (225 and 221 unique sequences in each pool,

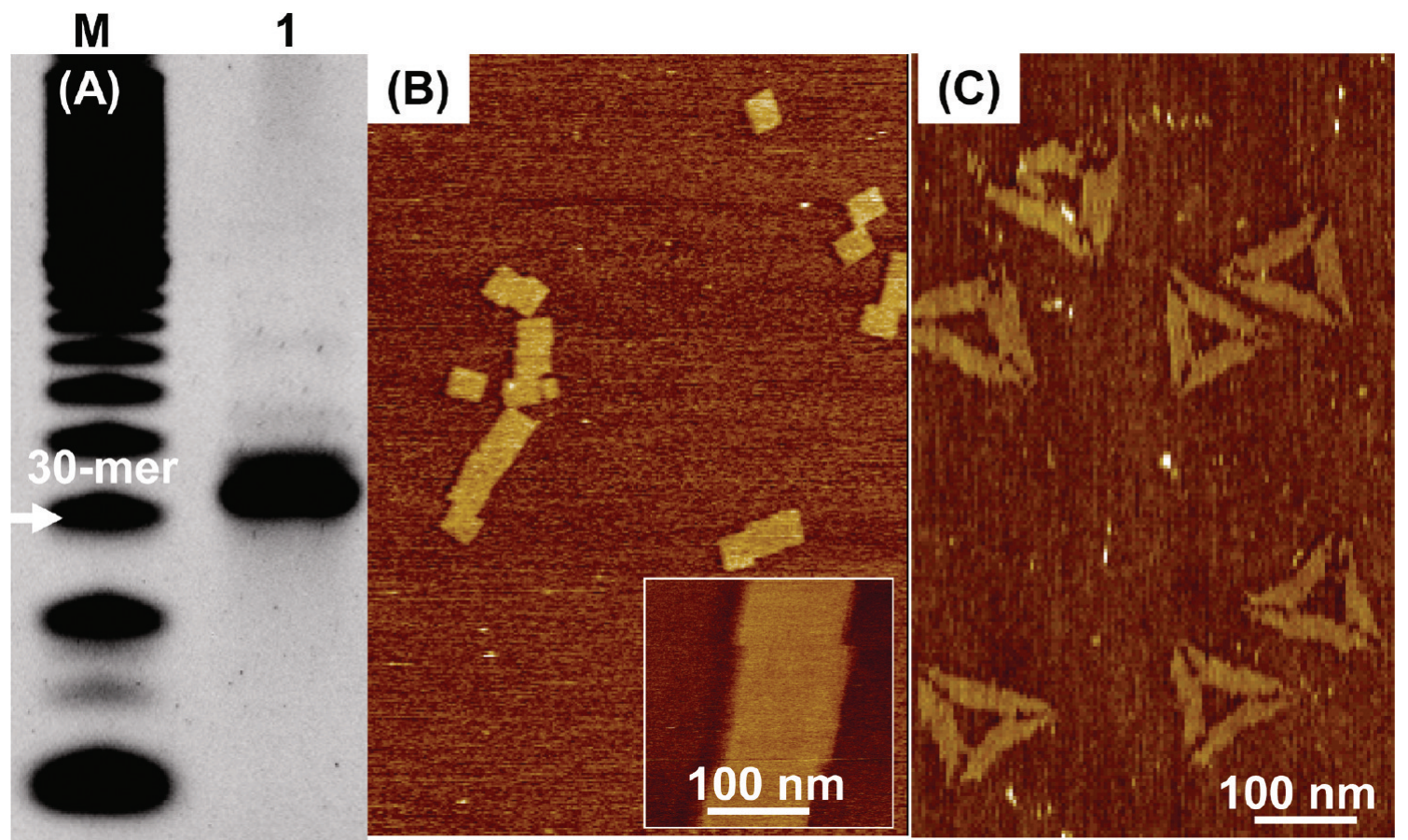

FIGURE 6. Construction of DNA origami with mixed-oligo pools synthesized and cleaved from silanized $\mathrm{SiO}_{2}-\mathrm{COC}$ slides. (A) Gel electrophoresis analysis of the released oligo pool of 225 unique oligos. Lane M, 10-bp ladder. (B) AFM image of rectangular DNA origami nanostructures formed by oligo pools released from $\mathrm{SiO}_{2}-\mathrm{COC}$ slides. A pool of 225 slide-derived oligos were used as "staple strands" for folding single-stranded M13 to form rectangular nanostructures. (C) AFM image of triangular DNA origami nanostructures formed by oligo pools released from $\mathrm{SiO}_{2}-\mathrm{COC}$ slides. Another batch of slide-derived oligos, comprising 221 unique sequences, was used to form triangular nanostructures. 
respectively; see the Supporting Information.), we were able to form tall rectangles and sharp triangles as shown in images B and C in Figure 6. The structures shown on AFM images appeared comparable to those presented by Rothemund, who used oligos individually synthesized by a standard column-based, solid-phase synthesis (46).

\section{DISCUSSION}

To our knowledge, this is the first report of in situ synthesis of oligonucleotide microarrays on COC substrate. In this study, we fabricated patterned $\mathrm{SiO}_{2}-\mathrm{COC}$ slides by selective RF sputter deposition. The patterned $\mathrm{SiO}_{2}$ thin-film spots on the inert and hydrophobic COC background were capable of constraining liquids by differential wettability, thereby effectively forming an array of picoliter reactors where in situ DNA synthesis could take place. Using standard phosphoramidite chemistry and an inkjet DNA synthesizer, we were able to synthesize oligo microarrays on the $\mathrm{SiO}_{2}-\mathrm{COC}$ slides with uniform features and satisfactory hybridization specificity. When synthesized directly on native silica silanols, oligos remained stable under PCR thermocycling conditions. When a cleavable linker was used, the in situ synthesized oligos were able to be cleaved and eluted from the slides and used directly to form correct DNA origami structures.

Using patterned $\mathrm{SiO}_{2}-$ COC substrates for in situ DNA synthesis is not only convenient but provides a number of major advantages. The method unites the well-developed and versatile glass and silane chemistry with the durable and flexible COC plastic matrix without any compromise in their optical properties (43). The deposited silica can either be utilized directly or modified readily with any off-the-shelf silane. The COC substrate can be molded to form requisite microfluidics and microstructures $(50,51)$ to further leverage the synthesized oligos. The designs of the microdevices can be easily prototyped by simple hot-embossing or massfabricated in a cost-effective fashion by injection molding (22). Using $\mathrm{SiO}_{2}-\mathrm{COC}$ substrates, there would be negligible loss of optical properties and the microarray slides could be imaged using standard microarray scanners. Additionally, the $\mathrm{SiO}_{2}-\mathrm{COC}$ hybrid is able to withstand high temperature and harsh organic chemicals, thereby increasing the operating range and applications of resulting devices. As the method presented here is based on PVD, future materials that maybe more effective for in situ synthesis than silica could possibly also be deposited (52).

Hybridization density and the resulting signal profile plays an important role in microarray quality. The silica thin film on $\mathrm{COC}$ is compositionally akin to glass. We determined by measuring the yields of cleaved oligos that an oligo density of $0.1-0.2 \mathrm{pmol} / \mathrm{mm}^{2}$ (approximately $0.8-1.2$ molecules/ $\mu \mathrm{m}^{2}$ ) was achievable on $\mathrm{SiO}_{2}-\mathrm{COC}$ slides, which was comparable to typical glass slides. In addition, our use of prepatterned substrates minimizes droplet misalignment that leads to cumulative synthesis errors in areas of "shifting boundary" on glass substrates. The patterning results in features possessing a sharp edge and uniform signal level. The possible mitigation of synthesis errors using patterned substrates could lead to reduced errors in products assembled using the synthesized oligos.

We observed that the hybridization and staining background signals were very low, as had been noted by other analytical studies with COC (15). This observation suggested that COC exposed to harsh organic chemicals used in DNA synthesis retained the same resistance to nonspecific adsorption as COC used for spotted arrays. This opens up the possibility of using in situ synthesized oligo microarrays in ultrasensitive analysis, which requires nonambiguous fluorescence signals at low analyte levels. Additionally, as arrays without cleavable linkers are able to withstand cleavage and $\mathrm{PCR}$ reaction conditions, the in situ COC oligo arrays can be employed in applications requiring high heat, extreme $\mathrm{pH}$, and solid-phase PCR (53).

This study also explored the possibility of using chipderived oligos as "staple strands" for constructing DNA origami. Our successful formation of rectangular and triangular origami structures suggests that such chip-derived oligos could be used to enable very large origami projects (i.e., requiring thousands of staple strands) that have thus far been difficult because of prohibitive cost of oligo synthesis. Chip-derived oligos could also lead to potential applications in synthetic biology such as assembly of genes and genome constructs.

\section{CONCLUSIONS}

In summary, this study demonstrated that RF sputter deposition of $\mathrm{SiO}_{2}$ thin film could be used as an effective technique to functionalize COC surface. It further demonstrated that high-quality DNA arrays could be fabricated on patterned $\mathrm{SiO}_{2}-\mathrm{COC}$ slides. This method could potentially be used for fabrication of high-quality and low-cost plastic DNA microchips in general. As various microstructures can be easily and directly molded on COC substrates, this study could provide a basis for further developments to integrate microarray with microfluidics for a broad array of bioanalytical, biofabrication, and diagnostic applications.

Acknowledgment. This work was supported by a Beckman Young Investigator Award and a Hartwell Individual Biomedical Research Award to J.T. The DNA origami work was supported partially by a National Science Foundation grant to T.H.L. We thank Dr. William Reichert for critical reading of the manuscript.

Supporting Information Available: Paragraph S1 contains extended and additional methods; Table S2 contains oligonucleotide sequences used in the staining and hybridization experiments; Table S3 contains sequences of "staple strands" used for forming DNA origami (PDF). This material is available free of charge via the Internet at http://pubs.acs.org.

\section{REFERENCES AND NOTES}

(1) Dobson, M. G.; Galvin, P.; Barton, D. E. Exp. Rev. Mol. Diagn. 2007, 7, 359-370.

(2) Hoheisel, J. D. Nat. Rev. Genet. 2006, 7, 200-210.

(3) Lockhart, D. J.; Winzeler, E. A. Nature 2000, 405, 827-836.

(4) Shi, L.; Perkins, R. G.; Tong, W. The Current Status of DNA Microarrays. In Microarrays; Integrated Analytical Systems Series; Springer: New York, 2009; pp 3-24. 
(5) Tian, J.; Ma, K.; Saaem, I. Mol. BioSyst. 2009, 5, 714-722.

(6) Dahlgren, C.; Zhang, H. Y.; Du, Q.; Grahn, M.; Norstedt, G.; Wahlestedt, C.; Liang, Z. Nucleic Acids Res. 2008, 36, e53.

(7) Richmond, K. E.; Li, M.-H.; Rodesch, M. J.; Patel, M.; Lowe, A. M.; Kim, C.; Chu, L. L.; Venkataramaian, N.; Flickinger, S. F.; Kaysen, J.; Belshaw, P. J.; Sussman, M. R.; Cerrina, F. Nucleic Acids Res. 2004, 32, $5011-5018$

(8) Tian, J.; Gong, H.; Sheng, N.; Zhou, X.; Gulari, E.; Gao, X.; Church, G. Nature 2004, 432, 1050-1054.

(9) Zhou, X.; Cai, S.; Hong, A.; You, Q.; Yu, P.; Sheng, N.; Srivannavit, O.; Muranjan, S.; Rouillard, J. M.; Xia, Y.; Zhang, X.; Xiang, Q.; Ganesh, R.; Zhu, Q.; Matejko, A.; Gulari, E.; Gao, X. Nucleic Acids Res. 2004, 32, 5409-5417.

(10) Diaz-Quijada, G. A.; Peytavi, R.; Nantel, A.; Roy, E.; Bergeron, M. G.; Dumoulin, M. M.; Veres, T. Lab Chip 2007, 7, 856-862.

(11) Geissler, M.; Roy, E.; Diaz-Quijada, G. A.; Galas, J.-C.; Veres, T ACS Appl. Mater. Interfaces 2009, 1, 1387-1395.

(12) Li, Y.; Wang, Z.; Ou, L. M. L.; Yu, H.-Z. Anal. Chem. 2006, 79, 426433.

(13) Raj, J.; Herzog, G.; Manning, M.; Volcke, C.; MacCraith, B. D.; Ballantyne, S.; Thompson, M.; Arrigan, D. W. M. Biosens. Bioelectron. 2009, 24, 2654-2658.

(14) Sauer-Budge, A. F.; Mirer, P.; Chatterjee, A.; Klapperich, C. M.; Chargin, D.; Sharon, A. Lab Chip 2009, 9, 2803-2810.

(15) Zhao, Z.; Peytavi, R.; Diaz-Quijada, G. A.; Picard, F. J.; Huletsky, A.; Leblanc, E.; Frenette, J.; Boivin, G.; Veres, T.; Dumoulin, M. M.; Bergeron, M. G. J. Clin. Microbiol. 2008, 46, 3752-3758.

(16) Cretich, M.; Di Carlo, G.; Giudici, C.; Pokoj, S.; Lauer, I.; Scheurer, S.; Chiari, M. Proteomics 2009, 9, 2098-2107.

(17) Heyries, K. A.; Loughran, M. G.; Hoffmann, D.; Homsy, A.; Blum L. J.; Marquette, C. A. Biosens. Bioelectron. 2008, 23, 1812-1818.

(18) Situma, C.; Hashimoto, M.; Soper, S. A. Biomol. Eng. 2006, 23 213-231

(19) Srivannavit, O.; Gulari, M.; Hua, Z. S.; Gao, X. L.; Zhou, X. C. Hong, A. L.; Zhou, T. C.; Gulari, E. Sens. Actuators, B 2009, 140, 473-481.

(20) Bhattacharyya, A.; Klapperich, C. M. Anal. Chem. 2005, 78, $788-$ 792

(21) Jagannathan, N.; Papautsky, I. J. Micromech. Microeng. 2004, 14, 96-103

(22) Klapperich, C. M. Exp. Rev. Med. Devic. 2009, 6, 211-213.

(23) Wang, W.; Soper, S. A., Bio-MEMS: Technologies and Applications, 1st ed.; CRC: Boca Raton, FL, 2006; pp 117-141.

(24) Becker, H.; Gärtner, C. Anal. Bioanal. Chem. 2008, 390, 89-111.

(25) Lamonte, R. R.; McNally, D. Plast. Eng. 2000, 56, 51-55.

(26) Lamonte, R. R.; McNally, D. Adv. Mater. Processes 2001, 159, $33-$ 36 .

(27) Bedair, M. F.; Oleschuk, R. D. Anal. Chem. 2006, 78, 1130-1138.

(28) Gaudioso, J.; Craighead, H. G.J. Chromatogr., A 2002, 971, $249-$ 253
(29) Hu, S. W.; Ren, X. Q.; Bachman, M.; Sims, C. E.; Li, G. P.; Allbritton, N. L. Anal. Chem. 2004, 76, 1865-1870.

(30) Laib, S.; MacCraith, B. D. Anal. Chem. 2007, 79, 6264-6270.

(31) Mair, D. A.; Geiger, E.; Pisano, A. P.; Frechet, J. M. J.; Svec, F. Lab Chip 2006, 6, 1346-1354.

(32) Nikolova, D.; Dayss, E.; Leps, G.; Wutzler, A. Surf. Interface Anal. 2004, 36, 689-693.

(33) Rohr, T.; Ogletree, D. F.; Svec, F.; Frechet, J. M. J. Adv. Funct. Mater. 2003, 13, 264-270.

(34) Sun, Q. C.; Zhang, D. D.; Wadsworth, L. C.; Tappi, J. 1998, 81, $177-183$

(35) Tsao, C. W.; Hromada, L.; Liu, J.; Kumar, P.; DeVoe, D. L. Lab Chip 2007, 7, 499-505

(36) Bi, H.; Meng, S.; Li, Y.; Guo, K.; Chen, Y.; Kong, J.; Yang, P.; Zhong, W.; Liu, B. Lab Chip 2006, 6, 769-775.

(37) Fixe, F.; Dufva, M.; Telleman, P.; Christensen, C. B. V. Lab Chip 2004, 4, 191-195.

(38) Kimura, N. Biochem. Biophys. Res. Commun. 2006, 347, 477-484

(39) Kinoshita, K.; Fujimoto, K.; Yakabe, T.; Saito, S.; Hamaguchi, Y.; Kikuchi, T.; Nonaka, K.; Murata, S.; Masuda, D.; Takada, W.; Funaoka, S.; Arai, S.; Nakanishi, H.; Yokoyama, K.; Fujiwara, K.; Matsubara, K. Nucleic Acids Res. 2007, 35, e3.

(40) Lin, R.; Burns, M. A. J. Micromech. Microeng. 2005, 15, 2156 2162 .

(41) Nagino, K.; Nomura, O.; Takii, Y.; Myomoto, A.; Ichikawa, M.; Nakamura, F.; Higasa, M.; Akiyama, H.; Nobumasa, H.; Shiojima, S.; Tsujimoto, G. J. Biochem. 2006, 139, 697-703.

(42) Pu, Q.; Oyesanya, O.; Thompson, B.; Liu, S.; Alvarez, J. C. Langmuir 2007, 23, 1577-1583.

(43) Ma, K.-S.; Reza, F.; Saaem, I.; Tian, J.J. Mater. Chem. 2009, 19, $7914-7920$.

(44) Lausted, C.; Dahl, T.; Warren, C.; King, K.; Smith, K.; Johnson, M.; Saleem, R.; Aitchison, J.; Hood, L.; Lasky, S. Genome Biol. 2004, 5, R58.

(45) Saaem, I.; Papasotiropoulos, V.; Wang, T.; Soteropoulos, P.; Libera, M. J. Nanosci. Nanotechnol. 2007, 7, 2623-2632.

(46) Rothemund, P. W. Nature 2006, 440, 297-302

(47) Cohen, G.; Deutsch, J.; Fineberg, J.; Levine, A. Nucleic Acids Res. 1997, 25, $911-912$.

(48) Dufva, M. Biomol. Eng. 2005, 22, 173-184

(49) Garland, P. B.; Serafinowski, P. J. Nucleic Acids Res. 2002, 30, e99.

(50) Illa, X.; Malsche, W. D.; Bomer, J.; Gardeniers, H.; Eijkel, J.; Morante, J. R.; Romano-Rodriguez, A.; Desmet, G. Lab Chip 2009, 9, $1511-1516$

(51) Myers, F. B.; Lee, L. P. Lab Chip 2008, 8, 2015-2031.

(52) Phillips, M. F.; Lockett, M. R.; Rodesch, M. J.; Shortreed, M. R.; Cerrina, F.; Smith, L. M. Nucleic Acids Res. 2008, 36, e7.

(53) Adessi, C.; Matton, G.; Ayala, G.; Turcatti, G.; Mermod, J.-J.; Mayer, P.; Kawashima, E. Nucleic Acids Res. 2000, 28, e87.

AM900884B 